\title{
Evidence for dust accumulation just outside the orbit of Venus
}

\author{
C. Leinert ${ }^{1}$ and B. Moster ${ }^{1}$
}

\begin{abstract}
Max-Planck-Institut für Astronomie, Königstuhl 17, 69117 Heidelberg, Germany
e-mail: leinert@mpia.de
\end{abstract}

Received 20 April 2007 / Accepted 7 June 2007

\begin{abstract}
Aims. To contribute to the knowledge of dynamics of interplanetary dust by searching for structures in the spatial distribution of interplanetary dust near the orbit of Venus.

Methods. We study the radial gradient of zodiacal light brightness, as observed by the zodiacal light photometer on board the Helios space probes on several orbits from 1975 to 1979. The cleanest data result from Helios B (=Helios 2) launched in January 1976.

Results. With respect to the general increase of zodiacal light brightness towards the Sun, the data show an excess brightness of a few percent for positions of the Helios space probe just outside the orbit of Venus.

Conclusions. We consider this as evidence for a dust ring associated with the orbit of Venus, somewhat similar to that found earlier along the Earth's orbit.
\end{abstract}

Key words. space vehicles: instruments - interplanetary medium - techniques: photometric

\section{Introduction}

The orbital evolution of interplanetary dust with its typical sizes of $1 \mu \mathrm{m}$ to $100 \mu \mathrm{m}$ is dominated by the Poynting-Robertsoneffect. This braking effect resulting from the aberration of solar radiation drives the grains into the Sun in astronomically short times, about $10^{5}$ years for a $10 \mu \mathrm{m}$ particle originating at $3 \mathrm{AU}$ distance from the Sun in the asteroid belt (Wyatt \& Whipple 1950). Unless interplanetary dust is a transitory phenomenon of our times, this requires continuous replenishment. Most of the source region for interplanetary dust particles lies in the asteroid belt and beyond (Dermott et al. 2001). While spiralling inwards, these interplanetary dust particles will cross the orbits of the inner planets where gravitational perturbations of these planets may produce detectable signatures in the overall spatial distribution of interplanetary dust. Indeed, a circumsolar dust ring along the Earth's orbit producing an excess of about $10 \%$ in zodiacal light brightness was detected in the infrared zodiacal light measurements of IRAS and COBE (Reach et al. 2002). Numerical simulations (Dermott et al. 1994, 2001) have explained the main features by resonances experienced by particles in low-inclination orbits, probably originating from sources in the asteroid belt. It is expected that similar effects might be seen with the next inner planet, Venus. We therefore decided to search the zodiacal light data of the Helios space probes (Leinert et al. 1981a), which crossed the orbit of Venus, for a brightness excess of similar size which could show the presence of a density enhancement, or "dust ring" along the orbit of Venus. To our knowledge this is the only data set which adresses this issue. Such a study is appropriate now, whith the study of a Solar Probe mission into the outer solar atmosphere signaling renewed interest in the inner solar system. Also, the necessary knowledge of basics and details of the operation of the Helios space probes and their zodiacal light photometer instrument is still available.

\section{Instrument}

Helios is a spacecraft spinning with one revolution per s with the spin axis perpendicular to its orbital plane which is the ecliptic. The zodiacal light is measured by three photometers mounted rigidly into the spacecraft. One - the $90^{\circ}$-photometer - looks parallel to the spin axis towards the ecliptic pole with a circular filed-of-view of $3^{\circ}$ diameter. The other two - the $15^{\circ}$-photometer and the $30^{\circ}$-photometer - are mounted at angles such that they scan small circles of ecliptic latitudes $\beta=16^{\circ}$ and $\beta=31^{\circ}$ with instantaneous square fields-of-view of $1^{\circ} \times 1^{\circ}$ and $2^{\circ} \times 2^{\circ}$. For Helios A the three photometers were looking south, for Helios B they were looking north of the ecliptic (Leinert et al. 1975).

Observations were performed in the visual in the $U, B$, and $V$ bands. As detector a photon counting photomultiplier of type EMR 541N was used. An integration of the $90^{\circ}$-photometer lasted $126 \mathrm{~s}$. The signal of the $360^{\circ}$ scans performed by the $15^{\circ}$ - and $30^{\circ}$-photometers was integrated into 32 sectors of width $5.6^{\circ}, 11.2^{\circ}$ and $22.5^{\circ}$ over 513 revolutions, resulting in integration times per sector of $8 \mathrm{~s}, 16 \mathrm{~s}$ or $32 \mathrm{~s}$ respectively. The signals were strong, with typically $2 \times 10^{4}$ to $4 \times 10^{5}$ counted photoelectrons contributing to each of the individual measurements. For a concise description of the instrument see Leinert et al. (1975).

One of the strong points of the zodiacal light instrument is its stability. Apart from a slow trend related to the orbital temperature variation inside the spacecraft, the stability measured over a month on an internal calibration lamp (Leinert et al. 1981b) or on the sky (Richter et al. 1982) was better than $0.5 \%$ with respect to the smooth average trend, and the long-term stability over the whole 11 year lifetime of Helios A was about $0.1 \%$ per year (Leinert \& Pitz 1989). It is this stability which makes it worthwhile at all to search for effects of density structure in the interplanetary dust cloud near the orbit of Venus. By analogy to the Earth's resonant ring such structures - if they exist - are expected to result in brightness structures of only a few percent. 
Table 1. Gemometry of the crossings of Venus' orbit.

\begin{tabular}{lrrrc}
\hline \hline & $R(\mathrm{AU})$ & $\lambda\left(^{\circ}\right)$ & $z_{\mathrm{v}}(\mathrm{AU})$ & $\begin{array}{c}\text { Conditions for } \\
\text { observing excess }\end{array}$ \\
\hline Helios A & & & & \\
$\quad$ inbound & 0.717 & 126.4 & 0.033 & $\begin{array}{c}\text { unfavourable } \\
\text { favourable }\end{array}$ \\
outbound & 0.733 & 30.6 & -0.036 & \\
Helios B & & & & favourable \\
inbound & 0.719 & 160.2 & 0.042 & neutral \\
$\quad$ outbound & 0.721 & 67.9 & -0.006 & \\
\hline
\end{tabular}

\section{Orbit}

Helios A and Helios B were launched on December 10, 1974 and January 16, 1976 into elliptical heliocentric orbits with perihelia at $0.31 \mathrm{AU}$ and $0.29 \mathrm{AU}$, respectively. The aphelia, according to the launch dates, were at heliocentric ecliptic longitudes $\lambda$ of $87^{\circ}$ and $114^{\circ}$. While the Helios orbits closely follow the ecliptic plane, the orbit of Venus is inclined by $3.4^{\circ}$ with a line of nodes at $\Omega=75.5^{\circ}$. At this heliocentric longitude and again $180^{\circ}$ later - Helios will cross the orbital plane of Venus. However, these positions are not relevant for our study. What counts, is the times when Helios is close to the orbit of Venus. In practice, we identify as points of closest approach those positions where the Helios spacecraft are crossing the orbit of Venus. This leads to four crossings with the Venus orbit, two for each spacecraft, one on the inbound, one on the outbound part of the Helios orbit. Given the inclination of Venus' orbit, its distance $z_{\mathrm{V}}$ from the ecliptic - north counted positive will usually be non-negligible, and it will have different values for the different crossings. The relevant geometrical parameters are summarised in Table 1. A northern position of the orbit of Venus is favourable when searching a brightness enhancement with the north-looking zodiacal light photometers on Helios B, unfavourable for the south-looking photometers on Helios A.

The orbital period of the Helios spacecraft of $190 \mathrm{~d}$ and $185 \mathrm{~d}$ are shorter than the $225 \mathrm{~d}$ period of Venus, therefore the position of Venus in its orbit changes by about $-60^{\circ}$ from one orbit crossing of Helios to the same crossing an Helios orbit later. Indeed, for one of the crossings (Helios A inbound on orbit 3 on May 8, 1976) Venus was by chance in the field-of-view of sector 12 of the $15^{\circ}$ - photometer. Since the inbound and outbound crossings also differ by $\sim 100^{\circ}$ in $\lambda$, our measurements below, averaged over several orbits, will not refer to a special position with respect to Venus but to average conditions along the Venus orbit.

Not all of the Helios orbits could be used for our study. Because of increasing solar activity, only orbits before the second half of 1978 were sufficiently free of solar flare and interplanetary plasma induced effects (Leinert \& Pitz 1989). Parts of the first orbit were affected by attitude maneuvers which abruptly changed viewing direction and therefore observed brightness.

\section{Geometry}

The geometry for the $90^{\circ}$-photometers is simple. While Helios is crossing a density enhancement, the zodiacal light photometer will locally observe the effects of any excess dust in the column along the line of sight towards the ecliptic pole. For the $15^{\circ}$ - and $30^{\circ}$-photometers with their oblique lines-of-sight the geometry is more involved. The best chances to detect an enhancement will be for those sectors where the line-of-sight is oriented almost tangentially to the orbit of Venus. These are in the numbering of the instrument the sectors 12 and 21 , the centers of which are at $\lambda-\lambda_{\odot}= \pm 84.4^{\circ}$ (sector 12) and $\lambda-\lambda_{\odot}=\mp 84.4^{\circ}$ (sector 21). Here, the upper sign is valid for Helios A, the lower one for Helios B with its flipped spin and orientation. The zodiacal light brightness in these directions is $2-2.5$ times higher than at the north ecliptic pole, but the longer oblique light path through a density enhancement compensates for this dilution by contributions from distant dust.

\section{Data}

We concentrate our search on data from Helios B. Helios A has the disadvantage that its $90^{\circ}$ photometer is pointing into the outer regions of the Magallanic cloud and that - due to to the failed deployment of one of its two antennas - the spacecraft and with it the field-of-view, e.g. of the $90^{\circ}$ photometer is wobbling at each revolution with an amplitude of about $0.5^{\circ}$. Therefore no standard reduction was attempted on these data. They are quality data only in the innermost solar system part of the Helios orbit where the zodiacal light is up to 17 times brighter than when close to the Earth. Also, the wobble mentioned above increases the uncertainty of corrections for individual stars in the $15^{\circ}$ - and $30^{\circ}$-photometers.

Thus we have a distinct north-south gradient in the systematic reliability of the data: those obtained with the $90^{\circ}$ photometer on Helios B are clearly the best, and the main conclusions should be based on them. The other photometers on Helios B are more subject to uncertainties in corrections of individual stars and of galactic background. We included those data sets which were apparently taken under favourable conditions concerning the contributions of background and individual stars. The consistency of these results with the data of the $90^{\circ}$ photometer strongly adds to our findings. Data from Helios A were less reliable at the level of percent accuracy for the reasons given above. We show those two examples which were still taken under relatively good conditions. They appear broadly consistent with the results of Helios B. This is a weaker but nevertheless favourable contribution to the overall picture.

\section{Data reduction}

Calibration and data reduction are described in Leinert et al. (1981a,b) and are not repeated here. We take the data as they were reduced by the Helios team during the time of the mission and stored at the data center NSSDC. The quantity we use is the calibrated zodiacal light brightness given in S10 units obtained from the total signal by subtracting dark current (negligible), light scattered by the electrons of the interplanetary medium (small), the contributions of individual stars (mostly small, sometimes dominating) and from integrated starlight (never small, often major). In the $V$ band one S10 unit - the equivalent of one solar type star of magnitude 10 per square degree corresponds to $1.18 \times 10^{-8} \mathrm{~W} \mathrm{~m}^{-2} \mathrm{sr}^{-1} \mu \mathrm{m}^{-1}$ or $1200 \mathrm{Jy} / \mathrm{sr}$.

Helios data reduction is non-recursive. For the reduction, the data were used as observed without removing or smoothing or interpolation. The reduction is based on literature values for star brightnesses and stellar background brightnesses and was done with calibrations and transformations to the colour system of the zodiacal light photometer as determined in the laboratory. Incorrect input brightnesses from the catalogues used or errors in spacecraft attitude will show as disturbances in the data. The disadvantages of this method are obvious. The advantage is that the result is transparently close to what the raw observations show with little improving art applied. This facilitates using the data again, now three decades after the initial, authorised reduction. 


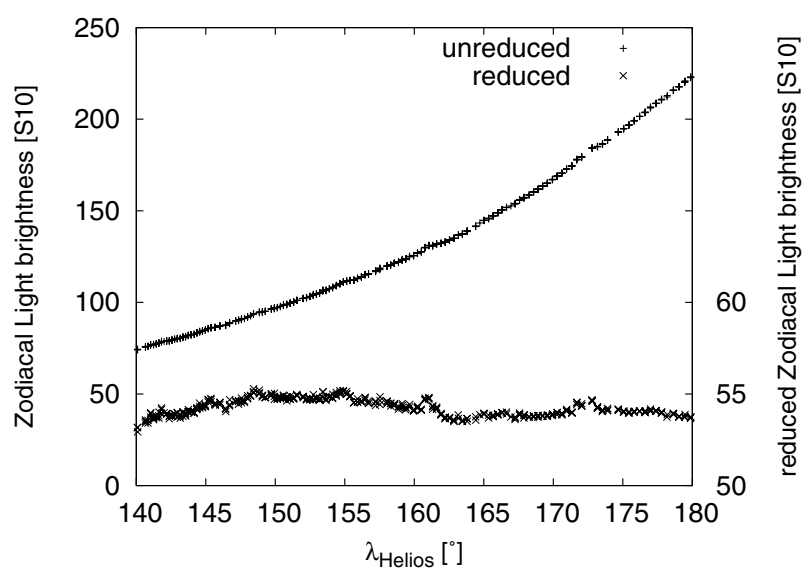

Fig. 1. Zodiacal light brightness (upper curve) and reduced brightness (lower curve) as seen with the $90^{\circ}$ photometer on Helios B in V during the inbound crossing of Venus' orbit in March 1977. The reduced brightness was obtained from the original results by removing the gradient (see text). The heliocentric longitude $\lambda_{\text {Helios }}$ gives the position of Helios along its orbit. Note the expanded scale with suppressed zero, which was used for the reduced brightness to show the remaining structure.

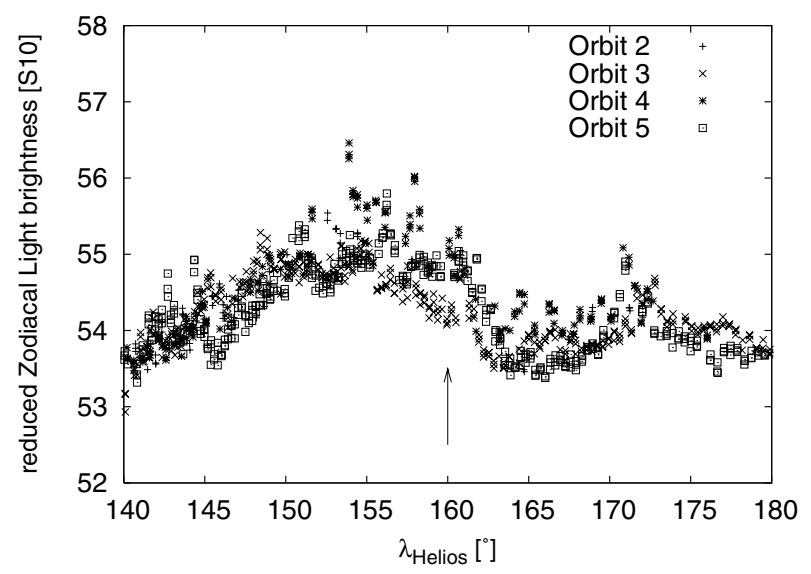

Fig. 2. Repeatability of the observed structure in reduced zodiacal light brightness from orbit to orbit of the Helios B space probe. The figure shows The $V$ band observations at the north ecliptic pole. The arrow indicates the position of Helios B at the inbound crossing of the orbit of Venus.

\section{Results}

The most obvious feature of Helios zodiacal light measurements is the strong brightness increase towards the Sun, approximately $\sim R^{-2.3}$, where $R$ is the Sun-Helios distance. Therefore, the data were multiplied by a factor $R^{\alpha}$ with exponent $\alpha$ close to 2.3 in order to produce a time series with little variation in which it would be easier to search for possible systematic variations near the orbit of Venus (Fig. 1).

In the following we always refer to this reduced brightness which has no precisely defined meaning but approximately gives the zodiacal light brightness at $1 \mathrm{AU}$. The multiplication by the smooth large-sale factor $R^{\alpha}$ does not introduce artefacts of measurable size. As noted above, the best data are available for Helios B.

We start with the results obtained with its $90^{\circ}$ photometer, since these - measuring locally the contribution from a column above the spacecraft - provide the most direct probe for enhancements in spatial density of interplanetary dust. These data do

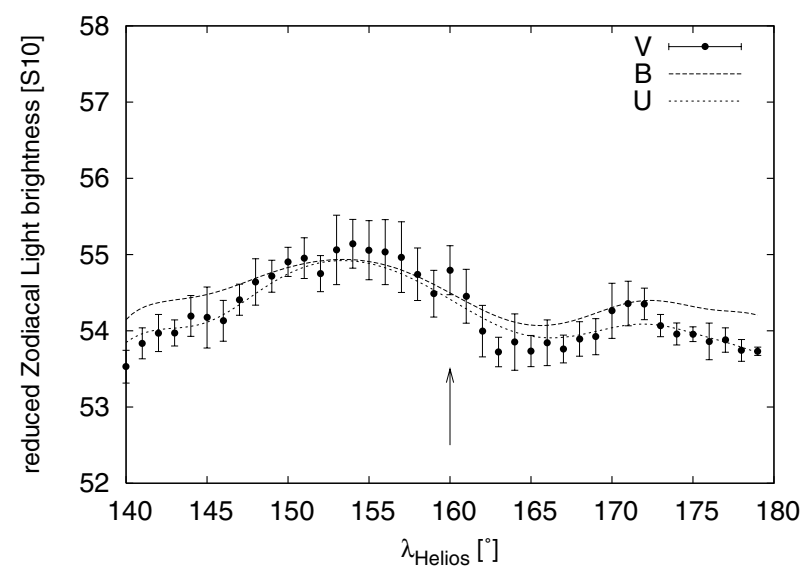

Fig. 3. Same as Fig. 2 but with the data averaged in $1^{\circ}$ intervals in heliocentric longitude, and with the observations in $U$ and $B$ superimposed. The error bars, shown for $V$ only, represent the rms scatter of the typically 10-20 measurements per bin. This is a quite conservative error, since for purely statistical fluctuations, the errors of the mean in each bin would be smaller by a factor of 3-4. Here, as in the following figures, the curves showing the averages of the $U$ and $B$ measurements have been shifted by one to a few S10 for ease of comparison, if necessary. The arrow marks the position of Helios B at the crossing.

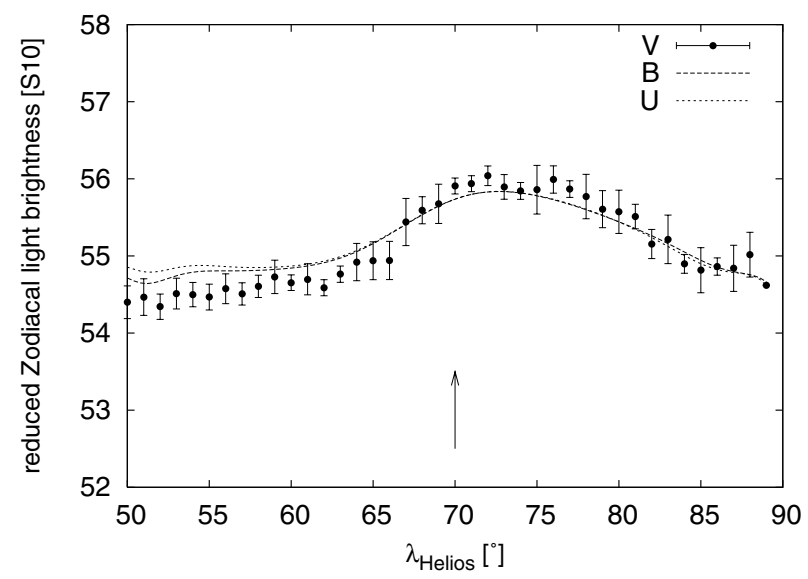

Fig. 4. Enhancement in reduced zodiacal light brightness near the outbound crossing of Venus' orbit as seen in the observations of Helios B towards the north ecliptic pole. Again, measurements from orbits 2-5 have been averaged. The arrow marks the position of Helios B at the crossing.

show a small brightness enhancement just outside the orbit of Venus, as suggested in Fig. 1. In the following we check the credibility of this effect.

If this brightness enhancement is due to a structure in the interplanetary dust cloud, one would expect it to repeat during each orbit at the same orbital position. Figure 2 shows for the observations towards the north ecliptic pole and for the inbound crossing that this is the case.

One would also expect that the enhancement has approximately solar colour, as is true for the zodiacal light. In other words this means that in $U, B$, and $V$ the brightness excess measured in $\mathrm{S} 10$ units should have the same size. This condition is also fulfilled as demonstrated in Fig. 3.

Finally, on the outbound crossing of Venus' orbit, - as expected - again an enhancement occurs just outside the orbit of Venus (Fig. 4). 

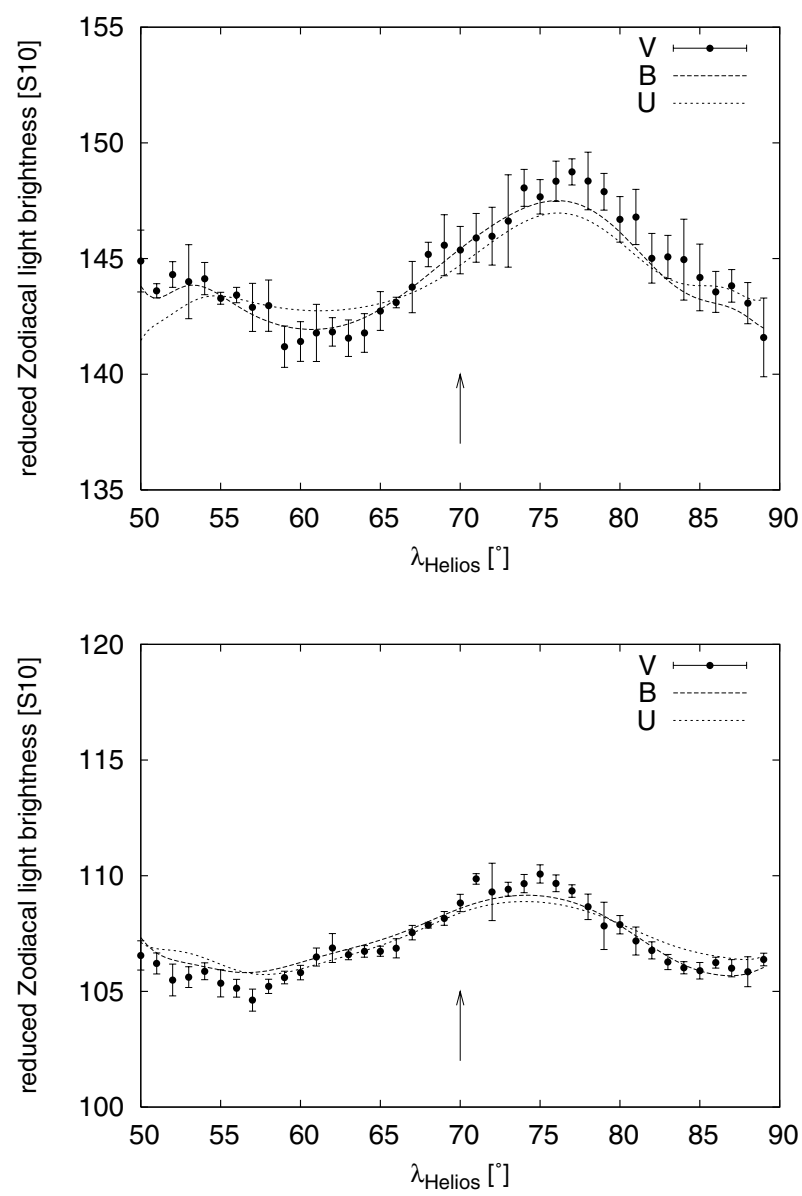

Fig. 5. Brightness enhancement seen with the other photometers of Helios B on the outbound crossing of Venus' orbit. Upper panel: sector 12 of the photometer looking at ecliptic latitude $\beta \approx 15^{\circ}$. Bottom: sector 12 of the photometer looking at ecliptic latitude $\beta \approx 30^{\circ}$. Same presentation as in Figs. 4 and 3, but the average is over orbits 1-5. The arrow marks the position of Helios B at the crossing.

It is virtually impossible that these effects are produced by stars passing through the field of view of the photometer. This field is always pointed towards the north ecliptic pole, with a small offset of $0.5^{\circ}$ which precesses along a circle around the ecliptic pole within one orbital period of Helios B, or with a motion of $0.09^{\circ}$ over the duration of the observed brightness enhancement. The excess brightness of about $2 \mathrm{~S} 10$ in the $3^{\circ} \mathrm{di}-$ ameter field-of-view could by produced by a star of magnitude $V=7.1$ with solar colour fully entering and leaving again the field-of-view at exactly the right times. No star this bright is present anywhere near the edge of the field-of-view, and even if it were, it could not move in and out again with such a small, one-directional displacement.

The $15^{\circ}$ - and $30^{\circ}$-photometers of Helios B are more prone to spurious brightness changes because of the varying stellar and diffuse background. However two of the eight combinations (two latitudes, two sectors, two crossings) both have low background and no bright stars in the field-of-view and therefore are expected to give reliable results. These, both referring to the outbound crossing of Venus' orbit, are shown in Fig. 5.

Figure 6 shows a counterexample. Here the brightness excess observed close to the orbit of Venus - as can be concluded from its colour - is mostly an artefact due to incomplete subtraction of stellar contributions. In this particular case it looks like the stellar excess signal due to the B1V star HR 1074 is

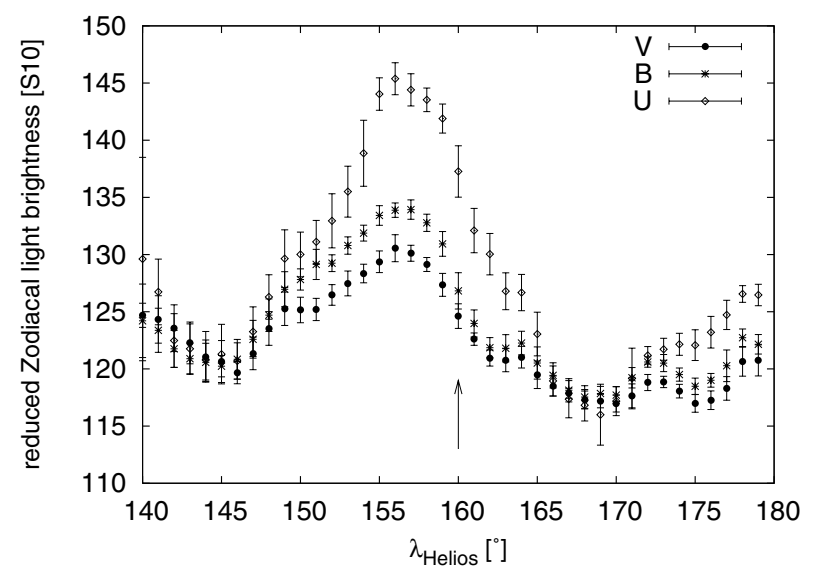

Fig. 6. Example of a false enhancement, observed by Helios B in sector 21 of the photometer looking at $\approx 15^{\circ}$ during the inbound crossing. The excess, rather blue, can be traced to the B1V star HR 1074.

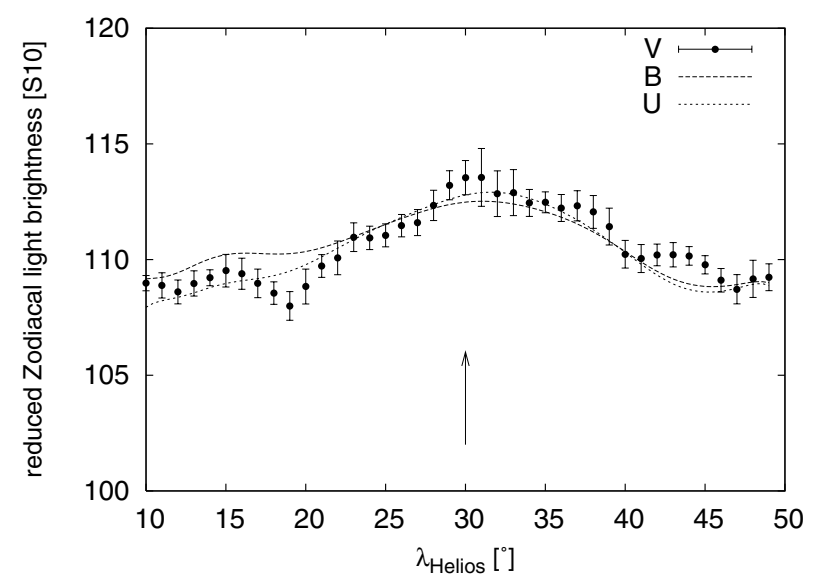

Fig. 7. Brightness enhancement seen with Helios $\mathrm{A}$ in sector 12 of the photometer looking at $\approx 30^{\circ}$ during the outbound crossing of Venus' orbit.

superimposed to a $\approx 4 \mathrm{~S} 10$ underlying zodiacal light brightening similar to that seen in Fig. 5. But a decomposition of the signal into these two contributions might be stressing the data. (With sufficient optimism one can guess the presence of a zodiacal light excess brightness signal also in the other disturbed data sets).

With Helios $A$ the conditions for detecting small brightness excesses are less favourable than for Helios B for the instrumental reasons mentioned above. In addition, there is a tendency for higher backgrounds and brighter stars in its south-looking photometers. The least interference by bright stars is for the outbound crossing shown in Fig. 7. There is also a measurement during an inbound crossing of Venus' orbit with low background (Fig. 8), but here the results for $V$ are somewhat discrepant from the results in $B$ and $U$. One could conclude that no zodiacal light enhancement in excess of $2 \mathrm{~S} 10$ is evident here.

In Table 2 we summarise the data sets with observed enhancements in zodiacal light brightness near the orbit of Venus and indicate why for other data sets reliable data could not be obtained.

\section{Discussion}

The results of the previous section, presented in Figs. 3-5, 7 and 8 , show a brightness enhancement by a few percent immediately outside the orbit of Venus. The interpretation depends on 


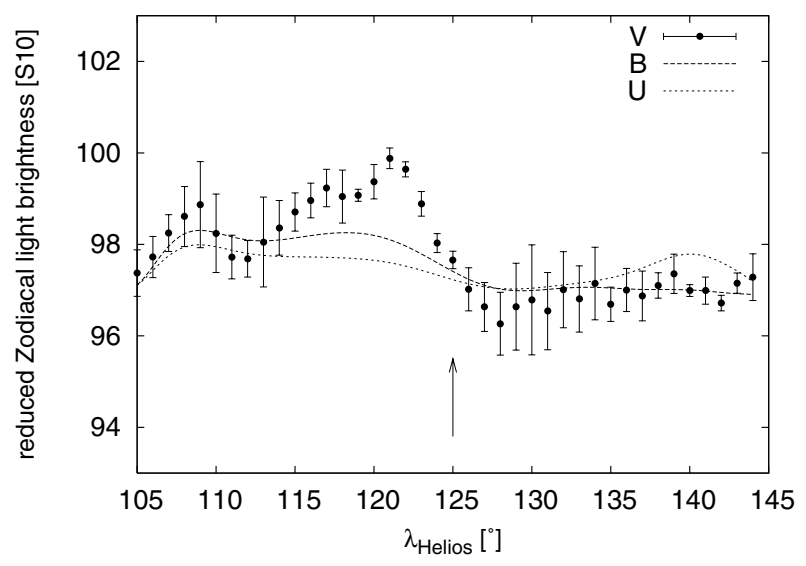

Fig. 8. Brightness variation seen with Helios A in sector 12 of the photometer looking at $\approx 30^{\circ}$ during the inbound crossing of Venus' orbit. There is some discrepancy between the measurements in the different colours, but at comparatively low level.

Table 2. Excesses in reduced zodiacal light brightness observed near the orbit of Venus.

\begin{tabular}{lllrlr}
\hline \hline Helios & Viewing & \multicolumn{3}{c}{ Inbound $\leftarrow$ Crossing $\rightarrow$ Outbound } \\
& direction & \multicolumn{1}{c}{$[\mathrm{S} 10]$} & $\%$ & {$[\mathrm{~S} 10]$} & $\%$ \\
\hline $\mathrm{B}$ & $90^{\circ}$ & $1.2 \pm 0.3$ & 2 & $1.2 \pm 0.3$ & 2 \\
& $31^{\circ}$ sec. 12 & high background & $3.6 \pm 0.8$ & 3.5 \\
& $31^{\circ}$ sec. 21 & $\alpha$ Per at edge of FoV & \multicolumn{2}{l}{ high background } \\
& $16^{\circ}$ sec. 12 & bright star $\epsilon$ Oph & \multicolumn{2}{l}{$5.8 \pm 1.0$} & 4 \\
& $16^{\circ}$ sec. 21 & bright star at edge FoV & bright star $\Theta$ Peg \\
\hline A & $-16^{\circ}$ sec. 12 & Mira, $\eta, \Theta$ Cet in FoV & \multicolumn{2}{l}{ high background } \\
& $-16^{\circ}$ sec. 21 & V and B inconsistent & \multicolumn{2}{l}{ Procyon in FoV } \\
& $-31^{\circ}$ sec. 12 & V and B inconsistent & \multicolumn{2}{l}{$3.7 \pm 0.9$} & 3.5 \\
& $-31^{\circ}$ sec. 21 & high background & \multicolumn{2}{c}{ high background } \\
\hline
\end{tabular}

whether or not one accepts the reasons given in favour of an origin of this enhancement by structures in the spatial distribution of interplanetary dust.

\subsection{Upper limits to an excess in dust density near the orbit of Venus}

Upper limits are the result of our study if the relation of the brightness excess to underlying dust structures is not accepted, but the brightness excess is attributed to some other nonspecified effect. Any brightness fluctuations due to dust structures then have to be significantly less than the observed effect. Therefore a safe upper bound to possible enhancements of dust density near the orbit of Venus can be derived from the observed brightness increases. These are $2 \%$ for a viewing direction at $\beta=+90^{\circ}, 4 \%$ for a viewing direction at $\beta=+16^{\circ}$ and longitude $\lambda-\lambda_{\odot}=84^{\circ}$, and $3.5 \%$ for a viewing direction at $\beta= \pm 31^{\circ}$ and longitude $\lambda-\lambda_{\odot}=\mp 84^{\circ}$. Using a result from the following subsection, this would correspond to less than $10 \%$ overdensity in any dust structure extending over about $0.05 \mathrm{AU}$ in direction perpendicular to the ecliptic plane.

We consider this as a useful, not easy to perform observational achievement, and we leave it to others to determine how discriminating these limits may be for descriptions of orbital evolution of interplanetary dust particles.

Indeed our original intent was to derive such an upper limit for the smoothness of the interplanetary dust cloud near the orbit of Venus. However, the data suggested the existence of a nonrandom feature.

\subsection{Simple model - dust ring outside the orbit of Venus}

A dust ring is the result of our study if the relation of the brightness increase to underlying dust structures is accepted. To this end we have to verify whether the different detections of a small brightness increase could be caused by one single spatial structure.

For this test a very simple model will suffice: a ring just ouside the orbit of Venus with radial extent $\Delta R$ and thickness $\Delta z$, symmetrical to the orbital plane of Venus, and in which the spatial density of interplanetary dust is increased by $x \%$ with respect to the smooth overall radial distribution.

The fact that the symmetry plane of interplanetary dust deviates from the ecliptic plane does not present a problem to this simple model but rather supports it. For the inner solar system, the parameters of this symmetry plane were determined as $i=$ $3.0 \pm 0.3^{\circ}, \Omega=87 \pm 4^{\circ}$ (Leinert et al. 1980), almost coincident with the orbital plane of Venus. This gives some physical justification to assuming a ring model with symmetry to Venus' orbital plane.

\subsubsection{Predictions from the simple model}

The radial extent $\Delta R$ follows from the duration of the event and the velocity of Helios along its orbit. The full duration was about $11^{\circ}$ in $\lambda_{\text {Helios }}$ corresponding to $\Delta R \sim 0.08$ AU. The $F W H M$ extent measures about $8^{\circ}$ in $\lambda_{\text {Helios }}$ corresponding to $\Delta R \sim 0.06 \mathrm{AU}$. This appears to be the same for all detections.

Values for $\Delta z$ and $x$ are most directly connected to the observations with the $90^{\circ}$-photometer, which shows an excess in reduced zodiacal light brightness of $1.2 \mathrm{~S} 10$ or $2 \%$. The local scattering power of interplanetary dust at $1 \mathrm{AU}$ and scattering angle $90^{\circ}$ was determined using quite general arguments from the brightness gradient in the ecliptic by Dumont (1973). Dumont \& Levasseur-Regourd (1978) noted that therefore for observations to the north ecliptic pole at $1 \mathrm{AU}, 1.8 \mathrm{~S} 10$ are contributed by scattering along the first $0.01 \mathrm{AU}$ of of the line-of-sight. If the radial distribution of interplanetary dust follows a power law, which is true to good approximation in the inner solar system, similarity relations hold and the same value of $1.8 \mathrm{~S} 10$ per now $0.01 \cdot R_{\text {Helios }} \mathrm{AU}$ also applies for the observed reduced brightnesses. We cannot determine the quantities $\Delta z$ and $x$ separately from our observations, but just assuming $x=10 \%$, a ring thickness of $\Delta z=0.048$ AU would be necessary to produce the observed excess brightness. The inbound crossing of Helios B occurs 0.04 AU south of Venus' orbit and therefore will observe the full $1.2 \mathrm{~S} 10$ of this excess when looking to the north ecliptic pole.

For the lines-of-sight of the $15^{\circ}$ - and $30^{\circ}$-photometer, with $\beta=16.2^{\circ}$ and $31.3^{\circ}$, the part of the line-of-sight crossing the ring of enhanced dust density has the length $\Delta s=\Delta z / \sin (\beta)=$ $0.17 \mathrm{AU}$, respectively 0.09 AU. Since the first one or two tenths of an $\mathrm{AU}$ along the lines-of-sight the scattering angle is almost constant, $\approx 90^{\circ}$, the expected excess brightness in these photometers is larger than that observed with the $90^{\circ}$-photometer by the factor $1 / \sin (\beta)$, resulting in predicted brightness enhancements of $4.3 \mathrm{~S} 10$ in the $15^{\circ}$ - and $2.3 \mathrm{~S} 10$ in the $30^{\circ}$-photometer.

Smaller values are predicted for crossings where the lineof-sight does not penetrate the full thickness of the assumed ring because the orbit of Helios cuts the ring or passes it on the wrong side. Thus for the outbound crossing of Helios B the brightness excess should be smaller by about a factor of two, and for the inbound crossing of Helios A, virtually no enhancement should be seen. This last prediction could be best checked with the data 
of the $90^{\circ}$-photometer of Helios A, which unfortunately are not useable for the technical reasons given above.

\subsubsection{Comparison with the results}

The observations during the outbound orbit crossing of Helios B provide a remarkable data set. Not only shows the $1.2 \mathrm{~S} 10$ excess seen in the $90^{\circ}$-photometer solar colour, but the independent observations with the independent $15^{\circ}$ - and $30^{\circ}$-photometers also show excesses, and not only in the same colour but also in the same length along the Helios orbit and almost in the brightness ratios expected for a circumorbital ring of increased spatial content.

The inbound crossing at first glance looks consistent, showing the same brightness excess. But at least for the simple ring model the observed excesses are too small, they should show at about twice the strength at the outbound crossing.

According to the orbital geometry given in Table 1, the south-looking photometers on Helios A should see no or almost no brightness excess near the orbit of Venus on the inbound crossing but should experience the full signal on the outbound part. Unfortunately, the data of the $90^{\circ}$-photometer, which would give the most direct result - are not useable here. From Fig. 7 we see an excess of similar size as for Helios B on the outbound crossing. The excess here seems to be located rather on than outside the orbit of Venus, but this crossing is also at a somewhat larger heliocentric distance than the others (Table 1). An inbound crossing of Helios $\mathrm{A}$ is shown in Fig. 8. Here the data in $V$ show a structure not present in the bands $B$ and $U$ and therefore do not really qualify as reliable measurements. Hence we can only state that the data appear not inconsistent with the predicted absence of a clear brightness excess for this crossing.

We do not have as convincing proof as we would like. However the evidence is best where the data are cleanest, namely for the pole-viewing $90^{\circ}$-photometer of Helios B. In addition, in view of the smallness of the effect and of the known difficulty to correct for the stellar contributions in the $15^{\circ}$ - and $30^{\circ}$-photometers our interpretation of the data is that the observed excesses are a real feature of the zodiacal light and that they provide reasonably strong evidence for the existence of a ring of enhanced dust content just outside the orbit of Venus.

\subsection{Future research}

It would be natural to supplement these observations by specific dynamical studies of the orbital evolution of interplanetary dust particles near the orbit of Venus. However, this is beyond the scope of this paper. We limit ourselves to provide the observational input, to which we feel qualified, and leave the theoretical part to the specialists.

A search for a dust enhancement near the orbit of Mercury was not attempted. The low mass of this planet and the strong solar irradiation, resulting in a much faster Poynting-Robertson drift would act to keep any Mercury-induced enhancement of dust density very small.

It would be an interesting task for a future inner solar system space probe with imaging capabilities to try to obtain a picture of the suggested dust ring which is more complete than was possible with the local measurements of the Helios space probes at a total of four orbital crossroads.

\section{Summary and conclusion}

We searched the data of the zodiacal light photometer on the Helios A and and in particular on the Helios B space probe for a brightness excess close to the orbit of Venus with the following results:

- we found a small excess, $2 \%$ towards the north ecliptic pole, which is repeatable from orbit to orbit. For the outbound crossing of Helios B, the excess fulfills all of the following criteria which should hold if it was due to a local enhancement in interplanetary dust: solar colour, same orbital extent for the observed ecliptic latitudes of $\beta=16^{\circ}, 31^{\circ}$, and $90^{\circ}$, and excess brightness varying with $1 / \sin (\beta)$.

- interpreted by a dust ring just outside the orbit of Venus and symmetrical to its orbital plane, the radial width of this ring would be $0.06 \mathrm{AU} F W H M$. Its height would be $0.048 \mathrm{AU}$ for an assumed overdensity of interplanetary dust in this ring of $10 \%$.

- this very suggestive evidence is somewhat weakened by the fact that the excesses on the outbound crossing of Helios B have the same strength as for the inbound crossing, contrary to the prediction of this very simple ring model. Obviously this simple ring model cannot be the complete story.

- the data for Helios A are less reliable, but appear consistent with the effects produced by such a dust ring.

All in all, the evidence is sufficiently convincing - maybe not beyond any doubt - to suggest the existence of a ring of enhanced dust content just outside the orbit of Venus. It will be worthwhile to study the implications of this finding for the dynamics of interplanetary dust particles and to compare to the already known dust ring along the Earth's orbit.

Acknowledgements. We thank very much the many colleagues from the Helios project, in particular Eckhart Pitz and Hartmut Link, the co-PIs of the zodiacal light photometer instrument. By building the instrument and spacecraft, by performing operations, data transfer and data reduction these many, working tediously over a long time, provided the basis for this work. We gratefully acknowledge the efforts of the National Space Science Data Center NSSDC, which kept the data from being lost, and we thank very much Bernie Jackson from UCSD who brought these data into easily useable format. In particular we want to mention H. Czech from Dornier System who designed the high voltage supplies of the zodiacal light photometer to be by a substantial factor more stable and more repeatable than specified - without this gift we could not even have thought of starting this study.

\section{References}

Dermott, S. F., Jayaraman, S., XU, Y. L., et al. 1994, Nature, 369, 719

Dermott, S. F., Grogan, K., Durda, D. D., et al. 2001, in Interplanetary Dust, ed. E. Grün, B. A. S. Gustafson, S. F. Dermott, \& H. Fechtig (Heidelberg, New York: Springer Berlin), 569

Dumont, R., 1973, Planet. Space Sci., 21, 2149

Dumont, R., \& Levasseur-Regourd, A.-C. 1978, A\&A, 64, 9

Leinert, Ch., \& Pitz, E. 1989, A\&A, 210, 399

Leinert, Ch., Link, H., Pitz, E., et al. 1975, Raumfahrtforschung, 19, 264

Leinert, Ch., Richter, I., Hanner, M., \& Pitz, E. 1980, A\&A, 82, 328

Leinert, Ch., Richter, I., Pitz, E., \& Planck, B. 1981a, A\&A, 103, 177

Leinert, Ch., Pitz, E., Link, H., \& Salm, N. 1981b, Space Sci. Instrum., 5, 257

Reach, T. W., Franz, B. A., Weiland, J. L., et al. 2002, Nature, 374, 521

Richter, I., Leinert, Ch., \& Planck, B. 1982, A\&A, 110, 115

Wyatt, S. P., \& Whipple, F. L. 1950, ApJ, 111, 134 